\title{
Combining Optical Phase Conjugation and Volterra Equalisation: a Novel Nonlinearity Compensation Scheme
}

\author{
Gabriele Liga $^{(1)}$, Gabriel Saavedra( ${ }^{(1)}$, Polina Bayvel(1) \\ (1) Optical Networks Group, Dept. of Electronic \& Electrical Engineering, University College London, \\ London WC1E 7JE, UK, gabriele.liga.11@ucl.ac.uk
}

Abstract A novel nonlinearity compensation scheme combining optical phase conjugation and Volterra equalisation is proposed and assessed. This hybrid approach outperforms both Volterra and optical phase conjugation schemes, individually applied, by over $4 d B$ in EDFA-amplified fibre links.

\section{Introduction}

Nonlinearity compensation (NLC) continues to be an important area of research in high-speed optical transmission. In recent years, optical phase conjugation (OPC) has been rediscovered as an effective NLC technique ${ }^{1,2}$. By conjugating the optical field at the mid-point of a transmission link, OPC is able to compensate for linear and nonlinear impairments over the entire transmitted bandwidth. To achieve perfect compensation, however, symmetry relative to the conjugation point in both power and dispersion profile is required. This condition is not fulfilled in systems employing lumped optical amplification such as the erbium doped fiber amplifier (EDFA). In such systems, the performance of OPC is substantially impaired ${ }^{3,4}$.

In alternative to optical NLC, digital NLC techniques can potentially undo signal-signal nonlinear effects within the receiver bandwidth, regardless of the nature of the optical link. However, this comes at the expense of an often large implementation complexity, especially as the NLC bandwidth increases. Multi-channel digital backpropagation $(\mathrm{DBP})^{5}$ is the most notable example of this issue. Volterra equalisers have been proposed as an alternative to DBP due to their potentially lower complexity and highly parallel structure ${ }^{5-7}$. However, Volterra equalisation reconstructs the optical field using a first-order approximation, which leads to a reduced NLC performance as the transmitted power is increased.

In this work, a novel NLC scheme combining OPC and a modified Volterra equaliser is proposed with the aim of overcoming the limitations of each of these two techniques.

\section{Volterra-assisted OPC}

In EDFA-amplified links, the effectiveness of the OPC to mitigate fibre nonlinearities is reduced. This phenomenon can be understood through a perturbation analysis of fibre nonlinearity in the presence of OPC. In this scenario, the first order regular perturbation term in frequency domain, and over the $x$ polarisation, is given by

$$
\begin{aligned}
& A_{1 x}(\omega)=i \gamma \frac{8}{9} \iint\left[\mathcal{S}_{x x x}^{*}\left(-\omega,-\omega_{1},-\omega_{2}\right)\right. \\
& \left.+\mathcal{S}_{y y x}^{*}\left(-\omega,-\omega_{1},-\omega_{2}\right)\right] \xi^{*}\left(\frac{N_{s}}{2}, \omega, \omega_{1}, \omega_{2}\right) \\
& \times F\left(N_{s}, \omega, \omega_{1}, \omega_{2}\right) d \omega_{2} d \omega_{1},
\end{aligned}
$$

where $\gamma$ is the nonlinearity coefficient of the fibre,

$$
\begin{aligned}
& \mathcal{S}_{x x x}\left(\omega, \omega_{1}, \omega_{2}\right) \triangleq S_{x}^{*}\left(\omega_{1}\right) S_{x}\left(\omega_{2}\right) S_{x}\left(\omega+\omega_{1}-\omega_{2}\right), \\
& \mathcal{S}_{y y x}\left(\omega, \omega_{1}, \omega_{2}\right) \triangleq S_{y}^{*}\left(\omega_{1}\right) S_{y}\left(\omega_{2}\right) S_{x}\left(\omega+\omega_{1}-\omega_{2}\right),
\end{aligned}
$$

are the signal kernels, and $S_{x}(\omega), S_{y}(\omega)$ are the transmitted signal spectra over two orthogonal polarisations $x$ and $y$, respectively. $\xi\left(\frac{N_{s}}{2}, \omega, \omega_{1}, \omega_{2}\right)$ is the so-called phased-array term accounting for the accumulation of nonlinear effects over $\frac{N_{s}}{2}$ fibre spans for a link with $N_{s}$ spans, whereas

$$
\begin{aligned}
& F\left(N_{s}, \omega, \omega_{1}, \omega_{2}\right) \triangleq \\
& =\frac{\left(e^{-i \beta_{2} \Delta \omega L_{s}} e^{-\alpha L_{s}}-1\right)\left(\alpha-i \beta_{2} \Delta \omega\right)}{\alpha^{2}+\beta_{2}^{2} \Delta \omega^{2}} \\
& +\frac{\left(e^{-i \beta_{2} \Delta \omega L_{s}}-e^{-\alpha L_{s}}\right)\left(\alpha+i \beta_{2} \Delta \omega\right)}{\alpha^{2}+\beta_{2}^{2} \Delta \omega^{2}},
\end{aligned}
$$

with $\Delta \omega \triangleq\left(\omega-\omega_{1}\right)\left(\omega_{1}-\omega_{2}\right) . \quad \alpha, \beta_{2}$ and $L_{s}$ are the attenuation coefficient, the group velocity dispersion, and the span length of the fibre system considered, respectively. The function $\xi^{*}\left(\frac{N_{s}}{2}, \omega, \omega_{1}, \omega_{2}\right) \cdot F\left(N_{s}, \omega, \omega_{1}, \omega_{2}\right)$ represents the third-order Volterra nonlinearity kernel for a fibre link of $N_{s}$ spans employing mid-link OPC. In Fig. 1, the nonlinearity kernels for a $10 \times 100 \mathrm{~km}$ standard single-mode fibre (SSMF) link without 


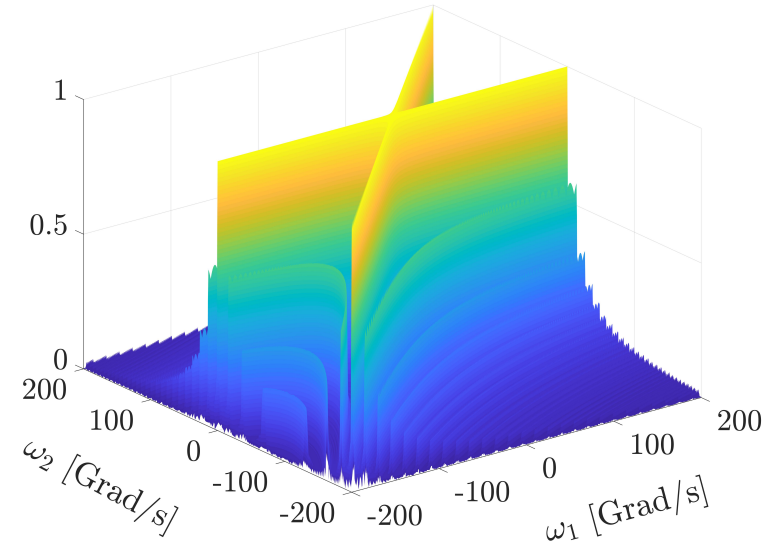

(a)

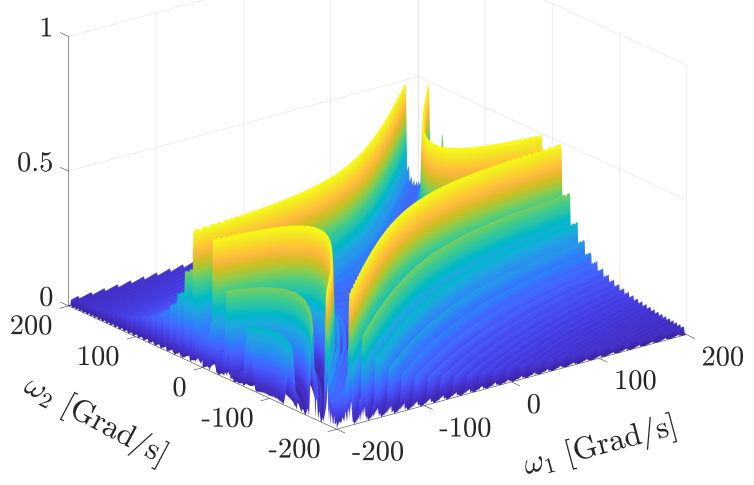

(b)

Fig. 1: Third-order nonlinearity kernels for a 10x100 km SSMF, EDFA-amplified link (a) without OPC, and (b) with OPC.

OPC (Fig. 1a) and with mid-link OPC (Fig. 1b) (kernel in Eq. (1)) are shown as a function of $\omega_{1}$, $\omega_{2}$, and for $\omega=0$. The kernels are normalised with respect to the maximum of the kernel without OPC (1a). It can be observed that the maximum value of the kernel in Fig. $1 \mathrm{~b}$ is reduced by a factor of 2 compared to its counterpart without OPC. Also, the OPC kernel shows an area of depression around the center of the 2D frequency domain, which indicates a strong NLC effectiveness for intra-channel nonlinear effects. However, Fig. 1 shows that coupling between higher frequency components is still significant even using OPC, which explains the reduction of the OPC NLC effectiveness in EDFA links. Eq. (1) corresponds to the third-order Volterra series term of fibre propagation in an EDFA-amplified link when OPC is used. As a result, a Volterra frequencydomain equaliser can be designed to compensate for this residual nonlinearity term, thus enhancing the performance of OPC. We call this approach Volterra-assisted OPC (VAO). Unlike a conventional Volterra equaliser, which is designed to undo the nonlinear term characterised by the kernel in Fig. 1a, the equaliser in the VAO scheme is designed to undo the perturbation in Eq. (1), characterised by the kernel in Fig. 1b.

\section{Nonlinearity Compensation Performance}

The VAO performance was assessed through numerical simulations of a $5 \times 32$ GBaud, $32.5 \mathrm{GHz}$ spacing, PM-16QAM root-raised cosine signal, transmitted over a $10 \times 100 \mathrm{~km}$ link using SSMF and EDFA amplification. As a figure of merit for the NLC effectiveness, we study the nonlinearity suppression factor, defined as

$$
\zeta=\frac{\sigma_{\mathrm{NLI}}^{2}}{\sigma_{\mathrm{NLC}}^{2}}
$$

where $\sigma_{\mathrm{NLI}}^{2}$ is the nonlinear interference (NLI) power in the absence of NLC, and $\sigma_{\mathrm{NLC}}^{2}$ is the residual NLI power after NLC is applied. $\zeta$ was calculated using optical noise-free (ideal amplification) simulations. Volterra equalisation for both the conventional Volterra scheme and VAO was applied to the entire transmitted 5-channel optical bandwidth. The equaliser structure was kept entirely non-recursive, i.e. a single integration for the entire optical link was performed. For complexity reasons, the equalisation was sequentially performed over adjacent blocks of 512 symbols each, within a sequence of $2^{16}$ symbols. An appropriate number of symbols was then discarded from each block to account for boundary effects.

The $\zeta$ factor as a function of transmitted power is shown in Fig. 2 for 3 different NLC schemes: mid-link OPC, a standard Volterra frequency domain equaliser (without OPC), and the proposed VAO. The results show that OPC and Volterra equalisation have approximately the same NLC effectiveness in EDFA-based systems. In particular, the $\zeta$ of the Volterra equaliser decreases with the transmitted power. This is the result of the first-order perturbative approximation and the imperfect cancellation of the nonlinear interference when applying Volterra over a limited block length. The OPC $\zeta$ is instead approximately constant across the entire range of powers shown in Fig 2. VAO significantly outperforms both OPC and conventional Volterra equalisation over the entire range of powers of in- 


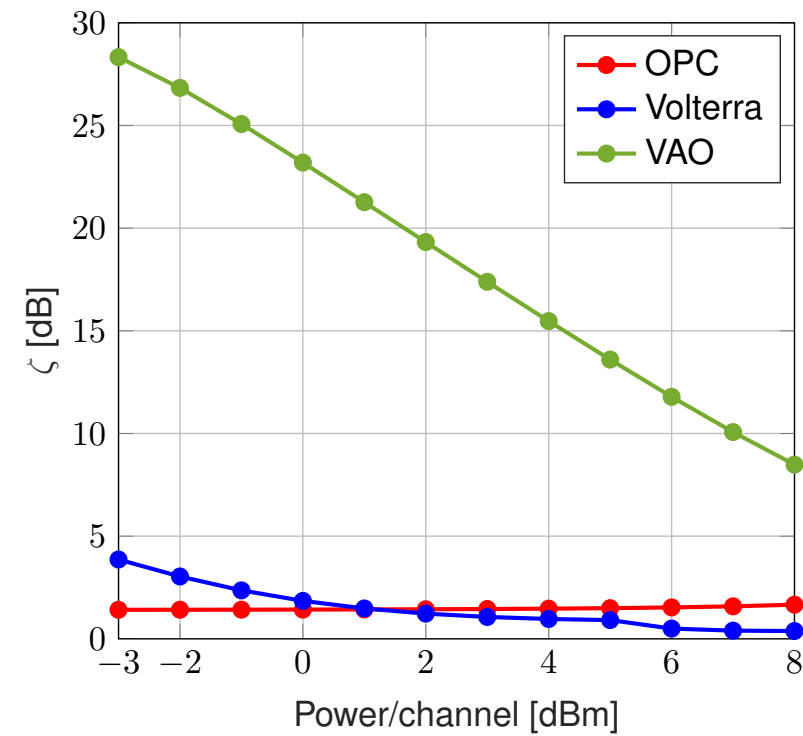

Fig. 2: Nonlinearity suppression factor as a function of transmitted power for the NLC schemes studied in this work.

terest. This can be attributed to the reduction of the NLI and its memory when OPC is applied. Both these effects are beneficial to the performance of the Volterra equaliser. It is interesting to notice that, the VAO $\zeta$ factor is significantly higher than the sum of the singular $\zeta$ contributions arising from OPC and conventional Volterra equalisation. This demonstrates the particular effectiveness of matching OPC with a Volterra equaliser. However, as the transmission power increases the VAO $\zeta$ still drops, similar to the conventional Volterra equaliser.

In order to evaluate the transmission performance using the VAO scheme, the received SNR in the presence of ASE noise was calculated for the system parameters previously considered. A noise figure of $5 \mathrm{~dB}$ was assumed for the EDFA amplifiers. Fig. 3 shows the SNR as a function of transmitted power for the 3 NLC approaches discussed in this paper and for an electronic dispersion compensation (EDC)-only receiver. Both Volterra and OPC show a gain of $0.4 \mathrm{~dB}$ compared to EDC. This surprisingly small SNR gain (considering the NLC bandwidth) is consistent with the results in Fig. 2, and confirms the limited effectiveness of both OPC and Volterra equalisation when individually used over the bandwidth considered in this study $(\approx 160 \mathrm{GHz})$. The enhanced NLC suppression given by the VAO scheme leads to an SNR gain of $4.6 \mathrm{~dB}$ over EDC, and $4.2 \mathrm{~dB}$ over either OPC or Volterra equalisation. Although the VAO effectiveness rapidly decays with transmitted power (see Fig. 2), the nonlinearity suppression is still sufficiently high for the powers of interest to yield a remarkable SNR

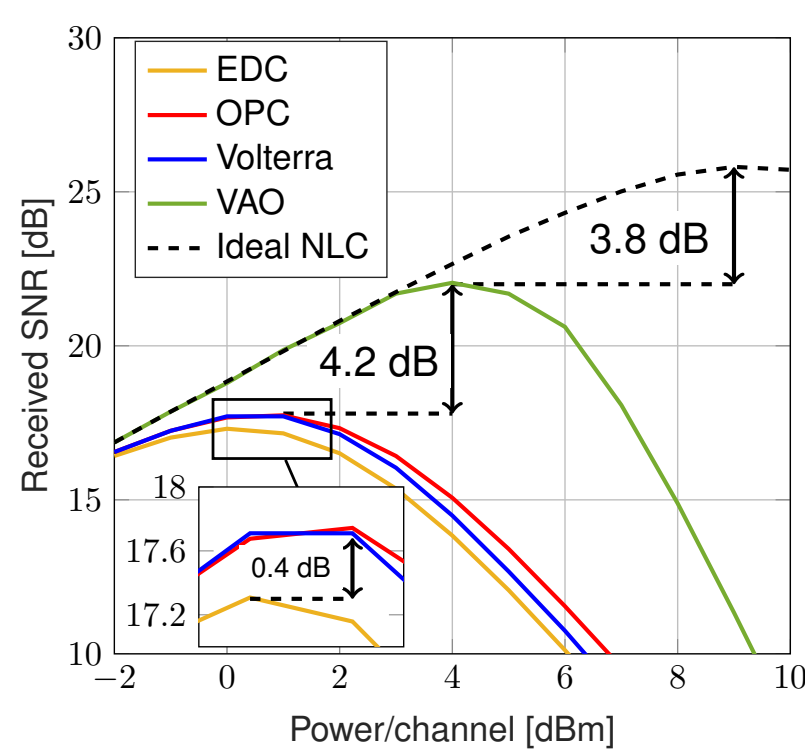

Fig. 3: SNR as a function of the transmitted power for the system considered in this work and different NLC schemes.

gain. Nonetheless, a $3.8 \mathrm{~dB}$ penalty relative to ideal nonlinearity compensation is still observed.

\section{Conclusions}

Jointly employing OPC and Volterra equalisation dramatically improves the nonlinearity compensation efficacy of each of these two schemes used individually. The Volterra-assisted OPC scheme, proposed in this work, achieves an SNR gain of $4.2 \mathrm{~dB}$ relative to either OPC- or Volterraonly schemes, in a 5-channel, $1000 \mathrm{~km}$, EDFAamplified optical link.

\section{Acknowledgements}

The UK EPSRC Programme Grants UNLOC EP/J017582/1 and INSIGHT EP/J017582/1 are gratefully acknowledged for the financial support.

\section{References}

[1] A. D. Ellis et al., "Capacity Limits of Systems Employing Multiple Optical Phase Conjugators," Opt. Express, Vol. 23, no. 16, p. 20381 (2015).

[2] L. B. Du, et al., "Fiber Nonlinearity Compensation for OFDM Super-Channels Using Optical Phase Conjugation," Opt. Express, Vol. 12, no. 18, p. 19921 (2012).

[3] V. Pechenkin et al. "Analysis of Four-Wave Mixing Suppression in Fiber-Optic OFDM Transmission Systems with an Optical Phase Conjugation Module," J. of Opt. Commun. and Network, Vol. 2, no. 9, p. 701 (2010).

[4] G. Saavedra et al., "Optical Phase Conjugation in Installed Optical Networks," Proc. OFC, W3E.2, San Diego (2018).

[5] J. C. Cartledge, et al., "Digital Signal Processing for Fiber Nonlinearities," Opt. Express, Vol. 25, no. 3, p. 1916 (2017).

[6] F. P. Guiomar et al., "Simplified Volterra Series Nonlinear Equalizer for Polarization-Multiplexed Coherent Optical Systems," J. Lightwave Technol., Vol. 31, no. 23, p. 3879 (2013).

[7] A. Bakhshali et al., "Frequency-Domain Volterra-Based Equalization Structures for Efficient Mitigation of Intrachannel Kerr Nonlinearities," J. Lightwave Technol., Vol. 34, no. 8, p. 1770 (2016). 